\title{
On a version of Trudinger-Moser inequality with Möbius shift invariance
}

\author{
Adimurthi \\ Centre of Applicable Mathematics \\ Tata Institute of Fundamental Research \\ P.O.Box No. 1234 \\ Bangalore - 560 012, India \\ aditi@aditi@math.tifrbng.res.in
}

\author{
Kyril Tintarev \\ Department of Mathematics \\ Uppsala University \\ P.O.Box 480 \\ SE-751 06 Uppsala, Sweden \\ kyril.tintarev@math.uu.se
}

May 29, 2018

\begin{abstract}
The paper raises a question about the optimal critical nonlinearity for the Sobolev space in two dimensions, connected to loss of compactness, and discusses the pertinent concentration compactness framework. We study properties of the improved version of the TrudingerMoser inequality on the open unit disk $B \subset \mathbb{R}^{2}$, recently proved by G. Mancini and K. Sandeep 13. Unlike the original Trudinger-Moser inequality, this inequality is invariant with respect to Möbius automorphisms of the unit disk, and as such is a closer analogy of the critical nonlinearity $\int|u|^{2^{*}}$ in the higher dimension than the original Trudinger-Moser nonlinearity.

2000 Mathematics Subject Classification. Primary 35J20, 35J60; Secondary 46E35, 47J30, 58J70.

Keywords. Trudinger-Moser inequality, elliptic problems in two dimensions, concentration compactness, weak convergence, Palais-Smale sequences.
\end{abstract}




\section{Introduction}

In this paper we study an inequality that improves the classical (Pohozhaev)Trudinger-Moser inequality ([10], [17], [9]) on a unit disk $B$ in $\mathbb{R}^{2}$ :

$$
\sup _{u \in H_{0}^{1}(B),\|\nabla u\|_{2} \leq 1} \int_{B} e^{4 \pi u^{2}} d x<\infty .
$$

The result below has been recently proved by Mancini and Sandeep [13] (for the invariant formulation in terms of hyperbolic space $\mathbb{H}^{2}$ see Theorem 2.3 below.)

Theorem 1.1. Let $B$ be an open unit disk in $\mathbb{R}^{2}$. The following relation holds true:

$$
\sup _{u \in H_{0}^{1}(B),\|\nabla u\|_{2} \leq 1} \int_{B} \frac{e^{4 \pi u^{2}}-1}{\left(1-|x|^{2}\right)^{2}} d x<\infty .
$$

We give a different proof to this inequality, based on coverings defined by Möbius transformations, rather than on rearrangements on the hyperbolic space like in [13], which provides insights for further results. An elementary corollary of this inequality is the Trudinger-Moser inequality in the exterior of the unit disk which immediately follows from (1.2) by the change of variable $x \mapsto x /|x|^{2}$.

Corollary 1.2. Let $B$ be an open unit disk in $\mathbb{R}^{2}$. The following relation holds true:

$$
\sup _{u \in \mathcal{D}_{0}^{1,2}\left(\mathbb{R}^{2} \backslash B\right),\|\nabla u\|_{2} \leq 1} \int_{\mathbb{R}^{2} \backslash B} \frac{e^{4 \pi u^{2}}-1}{\left(|x|^{2}-1\right)^{2}} d x<\infty .
$$

For the Sobolev space $H^{1}$ in two dimensions, the (Pohozhaev)-TrudingerMoser functional $\int e^{4 \pi u^{2}} d x$ is widely accepted as a standard nonlinearity of critical growth, that is, as a counterpart of $\int_{\mathbb{R}^{N}}|u|^{\frac{2 N}{N-2}} d x$ in the case $N>2$. This view is justified by the following analogy. When $N>2$, the functional $\int_{\mathbb{R}^{N}}|u|^{p} d x$ is continuous in $H^{1}\left(\mathbb{R}^{N}\right)$ when $p \in\left(2,2^{*}\right], 2^{*} \stackrel{\text { def }}{=} \frac{2 N}{N-2}$, and it is unbounded on any bounded subset of $H^{1}\left(\mathbb{R}^{N}\right)$ when $p>2^{*}$. In the case $N=2$, the functional $\int_{\mathbb{R}^{2}} e^{p u^{2}} d x$ on the set $\left\{u \in H^{1}\left(\mathbb{R}^{2}\right),\|u\|_{H^{1}} \leq 1\right\}$ is bounded if and only if $p \leq 4 \pi$ (see [12]). The analogy extends also to weak continuity properties. For obvious reason of translation invariance, there is no weak continuity if the domain of integration is the whole $\mathbb{R}^{N}$. If, however, 
$\Omega \subset \mathbb{R}^{N}, N>2$, is a bounded domain, then the functional $\int_{\Omega}|u|^{p} d x$ is weakly continuous on $H_{0}^{1}(\Omega)$ whenever $p<2^{*}$, and, similarly, if, $N=2$, the functional $\int_{\Omega} e^{p u^{2}} d x$ is weakly continuous on $\left\{u \in H_{0}^{1}(\Omega),\|u\|_{H_{0}^{1}} \leq 1\right\}$ whenever $p<4 \pi$.

This analogy, however, does not extend to the critical nonlinearities, $p=2^{*}$ resp. $p=4 \pi$. When $N>2$, the functional $\int_{\Omega}|u|^{2^{*}} d x$ is not weakly continuous at any point, but if $N=2$, the functional $\int_{\Omega} e^{4 \pi u^{2}} d x$ is sequentially weakly continuous at every point of $\left\{u \in H_{0}^{1}(\Omega),\|\nabla u\|_{2} \leq 1\right\} \backslash\{0\}$ (see [7]).

Lack of compactness for the critical nonlinearity in the case $N>2$ can be traced to the following symmetries of the space $\mathcal{D}^{1,2}\left(\mathbb{R}^{N}\right)$ (defined as the completion of $C_{0}^{\infty}$ with respect to the gradient norm $\left.\|\nabla \cdot\|_{2}\right)$ :

$$
D_{N}=\left\{g_{s, y} u(x)=2^{\frac{N-2}{2} s} u\left(2^{s}(x-y)\right), s \in \mathbb{R}, y \in \mathbb{R}^{N}\right\}
$$

that is, to actions of translations and dilations. These operators are linear isometries on both $\mathcal{D}^{1,2}\left(\mathbb{R}^{N}\right)$ and $L^{2^{*}}\left(\mathbb{R}^{N}\right)$, so that for every $u \in \mathcal{D}^{1,2}\left(\mathbb{R}^{N}\right)$, $u_{k} \stackrel{\text { def }}{=} g_{s_{k}, y_{k}} u \rightarrow 0$ whenever $\left|s_{k}\right|+\left|y_{k}\right| \rightarrow \infty$, while the respective norms of $u_{k}$ coincide with that of $u$. A similar counterexample suitable for a bounded domain is given by $y_{k}=0, s_{k} \rightarrow+\infty$ and $u$ supported on a convex compact subset. Lack of compactness in the imbedding of $\mathcal{D}^{1,2}\left(\mathbb{R}^{N}\right) \subset L^{2^{*}}\left(\mathbb{R}^{N}\right)$ can be, in some sense attributed entirely to the group $D_{N}$, namely, the compactness is restored if one "factors out" the action of the group (see e.g. Lemma 5.3, [16]):

$$
\forall s_{k} \in \mathbb{R}, y_{k} \in \mathbb{R}^{N}, g_{s_{k}, y_{k}} u_{k} \rightarrow 0 \text { in } \mathcal{D}^{1,2}\left(\mathbb{R}^{N}\right) \Rightarrow u_{k} \rightarrow 0 \text { in } L^{2^{*}}\left(\mathbb{R}^{N}\right) .
$$

Weak continuity properties of the critical nonlinearity in the case $N=2$ indicate that there is no known non-compact group, other than Euclidean shifts, that preserves both the Sobolev norm and the Trudinger-Moser nonlinearity $\int_{\mathbb{R}^{2}} e^{4 \pi u^{2}} d x$. The matter is further complicated by the fact that in this case there is no dilation-invariant functional space $\mathcal{D}^{1,2}$ : the completion of $C_{0}^{\infty}\left(\mathbb{R}^{2}\right)$ with respect to the gradient norm lacks continuous imbedding even into the space of distributions. On the other hand, the problem in the space $H_{0}^{1}(B)$ (which we in what follows consider equipped with the equivalent Sobolev norm $\|\nabla u\|_{2}$ ), admits two groups of linear unitary operators, defined below, that play a role similar, respectively, to actions of dilations and of translations. 
The Trudinger-Moser functional $\int_{B} e^{4 \pi u^{2}} d x$ fails to be invariant with respect to either of these groups. This, however, happens to testify not for irrelevance of these groups but for an observation that the Trudinger-Moser functional is not the sharp critical nonlinearity and can be replaced by a stronger expression. It remains an open problem, however, to find a sharp critical nonlinearity that is invariant with respect to the product group. The details are as follows.

\subsection{Dilation-invariant nonlinearity}

In this paragraph we summarize results of [3]. Let $H_{0, r}^{1}(B)$ denote the subspace of radial functions of $H_{0}^{1}(B)$. The transformations

$$
h_{s} u(r) \stackrel{\mathrm{d} e f}{=} s^{-\frac{1}{2}} u\left(r^{s}\right), u \in H_{0, r}^{1}(B) s>0
$$

preserve the norm $\|\nabla u\|_{2}$ of $H_{0, r}^{1}(B)$, as well as the 2-dimensional Hardy

functional $\int_{B} \frac{u^{2}}{|x|^{2}(\log 1 /|x|)^{2}} d x$ (for the Hardy inequality in dimension 2 see Adimurthi and Sandeep [2]). Furthermore, these transformation preserve the norms of a family of weighted $L^{p}$-spaces, $p=[2, \infty]$, analogous to the weighted- $L^{p}$ scale with $p \in\left[2,2^{*}\right]$ produced by Hölder inequality in the case $N>2$, interpolating between the Hardy term $\int \frac{u^{2}}{|x|^{2}} d x$ and the critical nonlinearity $\int|u|^{2^{*}} d x$. In the case $N=2$, the critical exponent is formally $2^{*}=+\infty$ and the dilation-invariant $L^{2^{*}}$-norm is

$$
\|u\|_{\infty}=\sup _{r \in(0,1)} \frac{|u(r)|}{\left(\log \frac{1}{r}\right)^{1 / 2}} .
$$

The Trudinger-Moser nonlinearity is not, however, dilation-invariant. On the other hand it is continuous with respect to the norm (1.5), which means that the $L^{\infty}$ - nonlinearity (1.5) gives a sharp, dilation-invariant improvement of the Trudinger-Moser nonlinearity, even if only for the subspace of radial functions.

\subsection{Möbius transformations}

We refer the reader to the Appendix for definitions and basic properties connected to Möbius transformations and the Poincaré disk. Adopting, for the sake of convenience, the complex numbers notation $z=x_{1}+i x_{2}$ for points 
$\left(x_{1}, x_{2}\right)$ on the plane, we consider the following subset of automorphisms of the unit disk, known as Möbius transformations.

$$
\eta_{\zeta}(z)=\frac{z-\zeta}{1-\bar{\zeta} z}, \zeta \in B
$$

Since the maps (1.6) are conformal automorphisms of the unit disk, one has $\left|\nabla u \circ \eta_{\zeta}\right|_{2}=|\nabla u|_{2}$, which implies that the Möbius shifts $u \mapsto u \circ \eta_{\zeta}$, $\zeta \in B$, are unitary operators in $H_{0}^{1}(B)$. The gradient norm on the disk is the coordinate representation of the quadratic form of Laplace-Beltrami operator on $\mathbb{H}^{2}$ regarded as the Poincaré disk model, which allows to identify $H_{0}^{1}(B)$ as representation of the space $\dot{H}^{1}\left(\mathbb{H}^{2}\right)$, defined by completion of $C_{0}^{\infty}\left(\mathbb{H}^{2}\right)$ with respect to the gradient norm. Möbius shifts give rise therefore to unitary operators on $\dot{H}^{1}\left(\mathbb{H}^{2}\right)$.

Furthermore, under the Poincaré disk model, the maps (1.6) define isometries on $\mathbb{H}^{2}$. Consequently, we have nonlinearities on the unit disk, invariant with respect to Möbius shifts, of the form (in the manifold notation and in the terms of Poincaré disk):

$$
\int_{\mathbb{H}^{2}} F(u) d \mu=\int_{B} F(u) \frac{d x}{\left(1-|x|^{2}\right)^{2}} .
$$

In particular, once the inequality (1.2) is verified, the functional $\int_{B} \frac{e^{4 \pi u^{2}}}{\left(1-|x|^{2}\right)^{2}} d x$ possesses both critical growth and invariance with respect to Möbius shifts.

\subsection{Main results}

In addition to Theorem 1.1, which trivially follows from its hyperbolic space counterpart, Theorem 2.3 proved in Section 2, we study weak continuity properties of subcritical (but not weakly continuous) nonlinearities of the form $\int_{B} F(u) \frac{d x}{\left(1-|x|^{2}\right)^{2}}$, and existence of maximizers for a related isoperimetric problem. We prove

Theorem 1.3. Let $F \in C(\mathbb{R})$ satisfy, with some $C>0, r>2$ and $p<4 \pi$,

$$
|F(s)| \leq C|s|^{r} e^{p s^{2}} .
$$

If $u_{k} \in H_{0}^{1}(B),\left\|\nabla u_{k}\right\|_{2}=1$, satisfies the condition

$$
\text { For every sequence } \zeta_{k} \in B, u_{k} \circ \eta_{\zeta_{k}} \rightarrow 0 \text {, }
$$


then

$$
\int_{B} F\left(u_{k}\right) d \mu \rightarrow 0
$$

This theorem is required for the following existence result.

Theorem 1.4. Let $F \in C^{1}(\mathbb{R})$, sup $F>0$, satisfy (1.7) with some $C>0$, $r>2$ and $p<4 \pi$. If, in addition, for every $t \in(0,1)$ and $a, b \in \mathbb{R}$,

$$
F\left(\sqrt{t a^{2}+(1-t) b^{2}}\right)>F(\sqrt{t} a)+F(\sqrt{1-t} b)
$$

then the maximum in

$$
M_{1} \stackrel{\text { def }}{=} \sup _{u \in H_{0}^{1}(B),\|\nabla u\|_{2}=1} \int_{B} F(u) d \mu
$$

is attained and for any minimizing sequence $u_{k}$ for (1.11) there exists a sequence $\zeta_{k} \in B$ such that $u_{k} \circ \eta_{\zeta_{k}} \rightarrow u \neq 0$. converges in $H_{0}^{1}(B)$ to the point of maximum.

In Section 3 we prove Theorem 1.3, Theorem 1.4, and a statement on the general structure of bounded sequences in $H_{0}^{1}(B)$, Theorem 3.2 , similar to Struwe's global compactness in [15]) and to a related statement of P.-L. Lions in [8] (note also that Möbius shifts are also involved in existence proof for the Plateau problem, [14]). In Section 4, the Appendix, we summarize relevant facts about the Poincaré disk.

\section{Proof of the invariant Trudinger-Moser in- equality}

$W \Subset B$ We start with the following elementary lemma.

Lemma 2.1. Let $W \subset \mathbb{R}^{2}$ be an open disk of radius $\frac{1}{2}$ and let

$$
\|u\|_{W}^{2} \stackrel{\text { def }}{=} \int_{W}\left(|\nabla u|^{2}+\lambda u^{2}\right) d x, \lambda>0
$$

There exists a number $q>0$ such that

$$
\sup _{u \in H^{1}(W),\|u\|_{W} \leq 1} \int_{W} e^{q u^{2}} d x<\infty .
$$


Proof. Let $T$ be an extension operator from $H^{1}(W)$ into $H_{0}^{1}(B)$. Then (2.2) follows from

$$
\sup _{u \in H_{0}^{1}(B),\|\nabla u\|_{2} \leq T \|} \int_{B} e^{q u^{2}} d x<\infty,
$$

which follows from the Trudinger-Moser inequality whenever $q \leq 4 \pi /\|T\|^{2}$

Lemma 2.2. Let $W \subset \mathbb{R}^{2}$ be an open disk of radius $\frac{1}{2}>0$ and let

$$
\|u\|_{W}^{2} \stackrel{\text { def }}{=} \int_{W}\left(|\nabla u|^{2}+\lambda u^{2}\right)^{2} d x, \lambda>0 .
$$

Let $q$ be as in Lemma 2.1. Then there is a positive constant $C=C(\lambda)$ such that for all $u \in H^{1}(W)$ satisfying $\|u\|_{W}<1$,

$$
\int_{W}\left(e^{q u^{2}}-1\right) d x \leq C \frac{\|u\|_{W}^{2}}{1-\|u\|_{W}^{2}} .
$$

Proof. Form (2.2) we have

$$
\frac{(q)^{n}}{n !} \int_{W}\left(u /\|u\|_{W}\right)^{2 n} d x \leq C, u \in H^{1}(W) \backslash\{0\} n \in \mathbb{N},
$$

and thus

$$
\frac{(q)^{n}}{n !} \int_{W} u^{2 n} d x \leq C\|u\|_{W}^{2 n}, n \in \mathbb{N} .
$$

Adding the inequalities (2.7) over $n \in \mathbb{N}$ and taking into account the assumption $\|u\|_{W}<1$, we obtain (2.5).

Theorem 2.3. The following relation holds true:

$$
\sup _{u \in \dot{H}^{1}\left(\mathbb{H}^{2}\right),\|u\| \leq 1} \int_{\mathbb{H}^{2}}\left(e^{4 \pi u^{2}}-1\right) d \mu<\infty .
$$

Proof. Consider $\mathbb{H}^{2}$ as the Poincaré disk $B$. Let $W \subset B$ be an open disk or radius $\frac{1}{2}$ and set the following equivalent Sobolev norm on $W$

$$
\|u\|_{W}^{2} \stackrel{\text { def }}{=}=\int_{W}\left(|\nabla u|^{2}+\frac{u^{2}}{\left(1-|x|^{2}\right)^{2}}\right) d x .
$$


By Corollary 4.2 there is a countable set $Z \subset B$ and the number $M \in \mathbb{N}$ be such that the sets $\eta_{\zeta}(W), \zeta \in Z$, cover $B$ with multiplicity not exceeding $M$. Let us fix a function $u \in \dot{H}^{1}\left(\mathbb{H}^{2}\right)$ such that $\|u\|_{\dot{H}^{1}} \leq 1$ and define

$$
Z_{u} \stackrel{\text { def }}{=}\left\{\zeta \in Z:\left\|u \circ \eta_{\zeta}\right\|_{W}^{2} \geq \frac{q}{8 \pi}\right\}
$$

where $q$ is as in Lemma 2.1. It is easy to see that $Z_{u}$ contains at most $40 \pi M / q$ elements. Indeed, since the multiplicity of the covering of $B$ by $\eta_{\zeta} W, \zeta \in Z$, is not greater than $M$, taking into account that the $\dot{H}^{1}\left(\mathbb{H}^{2}\right)$ norm in the Poincaré disk model is realized by $\|\nabla u\|_{2}$, and applying the Hardy's inequality (4.1), we have

$$
\frac{q}{8 \pi}\left(\# Z_{u}\right) \leq \sum_{\zeta \in Z_{u}}\left\|u \circ \eta_{\zeta}\right\|_{W}^{2} \leq M\left(\left.\|u\|\right|_{\dot{H}^{1}} ^{2}+\int_{B} u^{2} d \mu\right) \leq\left. 5 M\|u\|\right|_{\dot{H}^{1}} ^{2} \leq 5 M .
$$

From Lemma 2.2, applied to $\sqrt{\frac{4 \pi}{q}} u$ we have, for every $\zeta \in Z \backslash Z_{u}$,

$$
\int_{\eta_{\zeta} W}\left(e^{4 \pi u^{2}}-1\right) d \mu \leq C \frac{\left\|u \circ \eta_{\zeta}\right\|_{W}^{2}}{\frac{q}{4 \pi}-\left\|u \circ \eta_{\zeta}\right\|_{W}^{2}} \leq \frac{8 \pi}{q} C\left\|u \circ \eta_{\zeta}\right\|_{W}^{2} .
$$

Adding the inequalities over $\zeta \in Z \backslash Z_{u}$ we obtain, using again the Hardy's inequality,

$$
\int_{\bigcup_{\zeta \in Z_{u}} \eta_{\zeta} W}\left(e^{4 \pi u^{2}}-1\right) d \mu \leq C \int_{B}|\nabla u|^{2} d x+C \int_{B} u^{2} d \mu \leq\left. C\|u\|\right|_{\dot{H}^{1}} ^{2} \leq C .
$$

On the other hand, from the usual Trudinger-Moser inequality for $u \circ \eta_{\zeta}$, with any $\zeta \in Z_{u}$, we have

$$
\int_{\eta_{\zeta} W}\left(e^{4 \pi u^{2}}-1\right) d \mu=\int_{W} e^{4 \pi\left(u \circ \eta_{\zeta}\right)^{2}} d \mu \leq C \int_{W} e^{4 \pi\left(u \circ \eta_{\zeta}\right)^{2}} d x \leq \int_{B} e^{4 \pi\left(u \circ \eta_{\zeta}\right)^{2}} d x \leq C .
$$

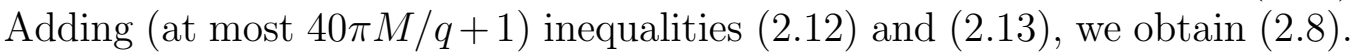

Proof of Theorem 1.2. Rewrite (2.8) in coordinate form for the Poincaré disk.

Remark 2.4. The constant $4 \pi$ in (2.8) as well as in (1.2) cannot be replaced by any number $p>4 \pi$. Indeed, the integrals in both relations are bounded from below by $\int_{B} e^{p u^{2}} d x$, from the Trudinger-Moser inequality, for which the parameter $4 \pi$ is optimal. 


\section{$3 \quad$ Existence of minimizers}

We begin with the proof of the first statement of Subsection 1.3.

Proof Theorem 1.3. Let us fix $p<4 \pi$. Let $u_{k} \in H_{0}^{1}(B),\left\|\nabla u_{k}\right\|_{2}=1$, and assume that $u_{k} \circ \eta_{\zeta_{k}} \rightarrow 0$ for every sequence $\zeta_{k} \in B$. By (2.8), there is a constant $C>0$ such that for all $n=0,1, \ldots$,

$$
\frac{p^{n}}{n !} \int_{B} u_{k}^{2 n} d \mu \leq C\left(\frac{p}{4 \pi}\right)^{n}
$$

Then, for every $m \in \mathbb{N}$ and for all $k \in \mathbb{N}$,

$$
\sum_{n \geq m} \frac{p^{n}}{n !} \int_{B} u_{k}^{2 n} d \mu \leq C\left(\frac{p}{4 \pi}\right)^{m}
$$

Furthermore, it is easy to see that there exists $\lambda \in(p / 4 \pi, 1)$ such that

$$
\sum_{n \geq m} \frac{p^{n}}{n !} \int_{B} u_{k}^{2 n+r} d \mu \leq C \lambda^{m}
$$

By Lemma 9.4 of [16], for every $n=0, \ldots, m-1$,

$$
\frac{p^{n}}{n !} \int_{B} u_{k}^{2 n+r} d \mu \rightarrow 0
$$

Combining (3.4) with (3.3), we obtain

$$
\limsup _{k \rightarrow \infty} \int_{B} F\left(u_{k}\right) d \mu \leq C \limsup _{k \rightarrow \infty} \int_{B}\left|u_{k}\right|^{r} e^{p u_{k}^{2}} d \mu \leq \epsilon,
$$

and since $\epsilon$ is an arbitrary positive number, (1.9) follows.

We will need the following version of Brezis-Lieb lemma in presence of a $H_{0}^{1}$-bound on a sequence.

Lemma 3.1. Let $F \in C^{1}(\mathbb{R})$ satisfy $|F(s)| \leq C s^{2} e^{p s^{2}}$ with some $C>0$ and $p<4 \pi$, and assume that $u_{k} \in H_{0}^{1}(B), u_{k} \rightarrow u$, $\left\|\nabla u_{k}\right\|_{2} \leq 1$. Then

$$
\int_{B}\left(F\left(u_{k}\right)-F\left(u_{k}-u\right)-F(u)\right) d \mu \rightarrow 0 .
$$


Proof. The notation of norm in this proof refers to the gradient norm $\|\nabla u\|_{2}$ on $B$. Note that $u_{k} \rightarrow u$ almost everywhere in $B$ and that lim sup $\left\|u_{k}-u\right\|^{2}=$ $\lim \sup \left\|u_{k}\right\|^{2}-\|u\|^{2} \leq 1$. Let $M>0$ and define

$$
F_{M}(s) \stackrel{\text { def }}{=}\left\{\begin{array}{cl}
F(s) & \text { for }|s|>M \\
0 & \text { for }|s| \leq M
\end{array}\right.
$$

Set $G_{M}=F-F_{M}$. Then $G_{M}$ is a bounded function and therefore, by Lebesgue convergence theorem,

$$
\int_{B}\left(G_{M}\left(u_{k}\right)-G_{M}\left(u_{k}-u\right)-G_{M}(u)\right) d \mu \rightarrow 0 .
$$

Fix two numbers $q, r$ such that $p<q<r<4 \pi$ and note that $\left|F_{M}(s)\right| \leq$ $s^{2} e^{q s^{2}} e^{-(q-p) M^{2}} \leq C\left(e^{r s^{2}}-1\right) e^{-(q-p) M^{2}}$. Then

$$
\left|\int F_{M}\left(u_{k}\right) d \mu\right| \leq C e^{-(q-p) M^{2}} \int_{B}\left(e^{r u_{k}^{2}}-1\right) d \mu \leq C e^{-(q-p) M^{2}}
$$

by (1.2), with analogous estimates when $u_{k}$ is replaced by $u$, resp. $u-u_{k}$. In the latter case (1.2) is applied to $u-u_{k}$ if $u_{k} \rightarrow u$ in norm, and to $\left(u-u_{k}\right) /\left\|u-u_{k}\right\|$ otherwise. From here and (3.7) we conclude that

$$
\limsup \left|\int_{B}\left(F\left(u_{k}\right)-F\left(u_{k}-u\right)-F(u)\right) d \mu\right| \leq C e^{-(q-p) M^{2}} .
$$

Since the number $M$ is arbitrary, (3.6) follows.

Proof of Theorem 1.4. For the length of this proof the notation of norm, unless otherwise specified, refers to the gradient norm $\|\nabla \cdot\|_{2}$. Let $u_{k} \in H_{0}^{1}(B)$ be such that $\left\|u_{k}\right\| \rightarrow 1$ and $\int_{B} F(u) d \mu \rightarrow M_{1}$. Consider the following family of problems that extends (1.11):

$$
M_{t} \stackrel{\text { def }}{=} \sup _{u \in H_{0}^{1}(B),\|u\|^{2}=t} \int_{B} F(u) d \mu, t \in[0,1] .
$$

If $u_{k}$ is a maximizing sequence then so is $u_{k} \circ \eta_{\zeta_{k}}$ for any sequence $\zeta_{k} \in B$. If $u_{k} \circ \eta_{\zeta_{k}} \rightarrow 0$ for any sequence $\zeta_{k}$, then by Theorem 1.3 we have $\int_{B} F\left(u_{k}\right) d \mu \rightarrow$ 0 , a contradiction since sup $F>0$ implies $M_{1}>0$. Thus we choose a sequence $\zeta_{k} \in B$ such that $u_{k} \circ \eta_{\zeta_{k}} \rightarrow u \neq 0$. 
By the standard scalar product calculations we have

$$
\|u\|^{2}+\left\|u_{k}-u\right\|^{2}=1
$$

while by Lemma 3.1,

$$
\int_{B} F(u) d \mu+\int_{B} F\left(u_{k}-u\right) d \mu \rightarrow M_{1} .
$$

Let $t=\|u\|$. Then from (3.11) follows

$$
M_{t}+M_{1-t} \geq M_{1} .
$$

An elementary argument using the well-known property of the gradient norm,

$$
\left\|v_{0}\right\| \leq 1,\left\|v_{1}\right\| \leq 1, v_{t}=\sqrt{t v_{1}^{2}+(1-t) v_{0}^{2}} \Rightarrow\left\|v_{t}\right\| \leq 1, t \in(0,1),
$$

shows that (3.12) contradicts (1.10) unless $t=1$ or $t=0$. The latter case has been, however, ruled out. Consequently, $\|u\|=1, \int_{B} F(u) d \mu=M$, and $u_{k} \rightarrow u$ in $H_{0}^{1}(B)$. The theorem is proved.

The notation $\stackrel{D}{\rightarrow} 0$ in the theorem below is a shorthand for the convergence in the sense of (1.8), which by Theorem 1.3 implies convergence in the sense of (1.9) and, in particular, convergence in $L^{p}(B, d \mu)$ for any $p \in[1, \infty)$.

Theorem 3.2. Let $u_{k} \in H_{0}^{1}(B)$ be a bounded sequence. Then there exists $w^{(n)} \in H, \zeta_{k}^{(n)} \in B, k, n \in \mathbb{N}$, such that for a renumbered subsequence

$$
\begin{gathered}
\zeta_{k}^{(1)}=0, \eta_{\zeta_{k}^{(n)}}\left(\zeta_{k}^{(m)}\right) \rightarrow \partial B \text { for } n \neq m, \\
w^{(n)}=\text { weak-lim } u_{k} \circ \eta_{\zeta_{k}^{(n)}}^{-1} \\
\sum_{n \in \mathbb{N}}\left\|\nabla w^{(n)}\right\|_{2}^{2} \leq \limsup \left\|\nabla u_{k}\right\|_{2}^{2} \\
u_{k}-\sum_{n \in \mathbb{N}} w^{(n)} \circ \eta_{\zeta_{k}^{(n)}} \stackrel{D}{\rightarrow} 0 .
\end{gathered}
$$

Proof. This theorem is an application of Theorem 3.1 in [16] to sequences in $\dot{H}^{1}\left(\mathbb{H}^{2}\right)$ equipped with the Möbius shifts. Conditions of that theorem have been verified for the case of actions of isometries on cocompact (or gridperiodic) manifolds, which includes hyperbolic spaces, in Lemma 2.9, [4]. Relation (3.13) is based on Remark 9.1 (a) of [16] 


\section{Appendix}

We summarize here some known definitions and facts concerning the Poincaré disk model of hyperbolic space. For reference see [5] and [11]. Poincaré disk is a coordinate representation of the hyperbolic space $\mathbb{H}^{2}$, consisting of the unit disk $B \subset \mathbb{R}^{2}$ equipped with the metric $g_{i, j}=\frac{1}{\left(1-|x|^{2}\right)^{2}} \delta_{i, j}, i, j=1,2$. The Riemannian measure $\mu$ on $\mathbb{H}^{2}$ is given in the Poincare disk model by $d \mu=\frac{d x}{\left(1-|x|^{2}\right)^{2}}$. The quadratic form of Laplace-Beltrami operator on $\mathbb{H}^{2}$ in the Poincaré disk model evaluates as $\int_{B}|\nabla u|^{2} d x$. The maps $\eta_{\zeta}: B \rightarrow B$, $\eta_{\zeta}(z)=\frac{z-\zeta}{1-\zeta z}, \zeta \in B$, are conformal isomorphisms of $\mathrm{B}$ as well as isometries of the Poincaré disk. Consequently, $\int_{B}|\nabla u|^{2} d x$ and $\int F(u) d \mu$ are preserved under transformations $u \mapsto u \circ \eta_{\zeta}$. The following version of Hardy's inequality holds true for all $u \in H_{0}^{1}(B)$, or, in invariant notations, $u \in \dot{H}^{1}\left(\mathbb{H}^{2}\right)$ (see [1] or [6]):

$$
\int_{B}|\nabla u|^{2} d x=\int_{\mathbb{H}^{2}}\left|\nabla_{\mathbb{H}^{2}} u\right|^{2} d \mu \geq \frac{1}{4} \int_{\mathbb{H}^{2}} u^{2} d \mu=\int_{B} u^{2} \frac{d x}{\left(1-|x|^{2}\right)^{2}} .
$$

The following lemma is well known (for example, it is a trivial modification of Lemma 2.3 from [4]). We give the proof of it for the sake of completeness.

Lemma 4.1. Let $U \Subset B$ be an open set, let

$$
V:=\bigcup_{\zeta \in B: \eta_{\zeta} U \cap U \neq \emptyset} \eta_{\zeta} U
$$

and let $W \Subset B$ be any open set, relatively compact in $B$, that contains $V$. There exist a number $M \in \mathbb{N}$, and a countable set $Z \subset B$ such that the sets $\left\{\eta_{\zeta} W\right\}_{\zeta \in Z}$, cover $B$ with multiplicity not exceeding $M$ and the sets $\left\{\eta_{\zeta} U\right\}_{\zeta \in Z}$ are mutually disjoint.

Proof. We show first that if $Z \subset B$ is a set such that the sets $\left\{\eta_{\zeta} U\right\}_{\zeta \in Z}$ are mutually disjoint, and the sets $\left\{\eta_{\zeta} W\right\}_{\zeta \in Z}$ cover $B$, then the latter collection has a uniformly finite multiplicity. Let $V_{r}(x)$ denote a geodesic ball of radius $r>0$ centered at $x \in B$, and note that $\mu\left(V_{r}(x)\right)$ is independent of $x \in B$. Let $R>0$ and $x_{0} \in B$ be such that $W \subset V_{R}\left(x_{0}\right)$. If $\eta_{\zeta} W$ intersects $\overline{V_{r}(x)}$, then $\eta_{\zeta} W \subset V_{r+2 R}(x)$; but since the sts $\eta_{\zeta} U, \zeta \in Z$, are disjoint, and $\eta_{\zeta} W \supset$ $\eta_{\zeta} V \supset \eta_{\zeta} U, \zeta \in Z$, that can be true for at most $\frac{\mu_{r+2 R}}{\mu(U)}$ values of $\zeta \in Z$. 
Therefore the number of $\zeta \in Z$ such that the set $\eta_{\zeta} W$ contains the point $x$ does not exceed $\frac{\mu_{r+2 R}}{\mu(U)}$.

Now let us construct the set $Z$.

Since every Riemannian manifold is paracompact, and once we observe that $\eta_{\zeta}(\{0\})=-\zeta$, so that $\bigcup_{\zeta \in B} \eta_{\zeta}(0)=B$, there exists a subset $Z_{0} \subset B$, such that $\left\{\eta_{\zeta} V\right\}_{\zeta \in B}$ is a locally finite cover of $B$. Indeed, we find first of all a locally finite refinement of the cover $\left\{\eta_{\zeta} V\right\}_{\zeta \in B}$, which via the refinement map determines a subcover, which also is locally finite due to the fact that all covering sets $\eta_{\zeta} V$ have the same finite geodesic diameter.

By induction we define subsets $Z_{k}=A_{k} \cup B_{k} \subset B$ such that the number of elements in $A_{k}$ equals $k$ and

$$
B=\bigcup_{\zeta \in A_{k}} \eta_{\zeta} V \cup \bigcup_{\zeta \in B_{k}} \eta_{\zeta} U
$$

and $\eta_{\zeta_{1}} U \cap \eta_{\zeta_{2}} U=\emptyset$ for any $\zeta_{1} \in A_{k}, \zeta_{2} \in Z_{k}, \zeta_{1} \neq \zeta_{2}$. Furthermore $A_{k} \subset$ $A_{k+1}$ for all $k$, while $B_{k} \supset B_{k+1}$ with $\cap_{k=0}^{\infty} B_{k}=\emptyset$. Since the cover $\left\{\eta_{\zeta} U\right\}_{\zeta \in Z_{0}}$ was locally finite, the latter implies that any compact set $K \Subset B$ is contained in $\bigcup_{\zeta \in A_{k}} \eta_{\zeta} V$ for sufficiently large $k$. Finally take $Z:=\bigcup_{k=0}^{\infty} A_{k}$. Begin with $A_{0}:=\emptyset, B_{0}:=Z_{0}$. Let $\left\{\zeta_{j}\right\}_{j \in \mathbb{N}}$ be an enumeration of $Z_{0}$. Assuming that $A_{k}, B_{k}$ have already been constructed, let us construct $A_{k+1}, B_{k+1}$. Let $j_{k}=\min \left\{j: \zeta_{j} \in B_{k}\right\}$. Set $A_{k+1}:=A_{k} \cup\left\{\zeta_{j_{k}}\right\}$ and let $B_{k+1}:=\{\zeta \in$ $\left.B_{k} ; \eta_{\zeta} U \cap \eta_{\zeta_{j_{k}}} U=\emptyset\right\}$.

Corollary 4.2. Let $W \Subset B$ be an open set. There exist a number $M \in$ $\mathbb{N}$, and a countable set $Z \subset B$ such that the sets $\left\{\eta_{\zeta} W\right\}_{\zeta \in Z}$ cover $B$ with multiplicity not exceeding $M$.

Proof. Let $\epsilon>0$ and $x \in W$ be such that the geodesic ball $V_{3 \epsilon}(x) \Subset W$. The corollary is immediate from Lemma 4.1 with $U=V_{\epsilon}(x)$ once we note that the set (4.2) is contained in $V_{3 \epsilon}(x)$. Indeed, let $y \in V$. Then there exist $\zeta \in Z$ and $z \in V_{\epsilon}(x) \cap V_{\epsilon}\left(\eta_{\zeta} x\right)$ such that $y \in \eta_{\zeta} V_{\epsilon}(x)$. Then, by the triangle inequality for the geodesic distance, $d(x, y) \leq d(x, z)+d\left(z, \eta_{\zeta} x\right)+d\left(\eta_{\zeta} x, y\right)<3 \epsilon$.

\section{References}

[1] Adimurthi, A. Sekar, Role of the fundamental solution in HardySobolev-type inequality, Proc. Roy. Soc. Edinburgh Sect. A 136 (2006) 1111-1130. 
[2] Adimurthi, K. Sandeep, Existence and non-existence of the first eigenvalue of the perturbed Hardy-Sobolev operator, Proc. Roy. Soc. Edinburgh Sect. A 132 (2002), 1021-1043.

[3] Adimurthi, J. M. do Ó, K. Tintarev, Cocompactness and minimizers for inequalities of Hardy-Sobolev type involving $N$-Laplacian, preprint

[4] K.-H. Fieseler, K. Tintarev, Semilinear elliptic problems and concentration compactness on non-compact Riemannian manifolds, J. Geom. Anal. 13 (2003), 67-75.

[5] S.Lang, SL2(R). Graduate Texts in Mathematics 105 Springer-Verlag 1975.

[6] G. Mancini, K. Sandeep, On a semilinear elliptic equation in $\mathbb{H}^{n}$, Ann. Scuola Norm. Sup. Pisa Cl. Sci. (5) 7 (2008), 1-37.

[7] P.-L. Lions, The concentration-compactness principle in the calculus of variations. The limit case II, Rev. Mat. Iberoamericana 1 (1985), 45-121.

[8] Lions, P.-L., Solutions of Hartree-Fock equations for Coulomb systems, Comm. Math. Phys. 109 (1987), 33-97.

[9] J.Moser, A sharp form of an inequality by N.Trudinger, Indiana Univ.Math.J. 20 (1971) 1077-1092

[10] S. I. Pohozhaev, The Sobolev imbedding in the case $p l=n$, Proc. Tech. Sci. Conf. on Adv. Sci. Research 1964-1965, Mathematics Section, 158170, Moskov. Energet. Inst., Moscow 1965.

[11] J. G. Ratcliffe, Foundations of Hyperbolic Manifods, 2nd ed., Graduate Texts in Mathematics 149, Springer-Verlag 2006.

[12] B. Ruf, A sharp Trudinger-Moser type inequality for unbounded domains in $\mathbb{R}^{2}$, J. Func. Anal. 219 (2005), 340-367

[13] G. Mancini, K. Sandeep, Moser-Trudinger inequality on conformal disks, preprint

[14] M. Struwe, Plateau's problem and the calculus of variations, Mathematical Notes 35, Princeton University Press (1988). 
[15] M. Struwe, A global compactness result for elliptic boundary value problems involving limiting nonlinearities, Math. Z. 187 (1984), 511-517.

[16] K.Tintarev, K.-H.Fieseler, Concentration compactness: functionalanalytic grounds and applications, Imperial College Press, 2007

[17] N. S. Trudinger, On imbeddings into Orlicz spaces and some applications, J. Math. Mech. 17 (1967), 473-483. 